\title{
Respostas fisiológicas de vacas em lactação à ventilação e aspersão na sala de espera ${ }^{1}$
}

\author{
Physiological responses of dairy cows to fan plus sprinkler in holding pen
}

\author{
Irineu Arcaro Júnior ${ }^{2}$ Juliana Rodrigues Pozzi Arcaro ${ }^{2}$ \\ Claudia Rodrigues Pozzi ${ }^{2}$ Claudia Del Fava ${ }^{3}$ Helena Fagundes $^{4}$ \\ Soraia Vanessa Matarazzo ${ }^{5}$ Jean Eduardo De Oliveira $^{6}$
}

RESUMO

O objetivo deste trabalho foi avaliar a eficiência do uso da climatização no ambiente da sala de espera (ventilação forçada ou ventilação forçada e aspersão) sobre as variáveis ambientais e fisiológicas de vacas em lactação. O período experimental teve duração de 90 dias, em que foram avaliadas 21 fêmeas, com produção média de leite de $21 \mathrm{~kg}$ leite $d^{-1}$,distribuídas em delineamento inteiramente casualizado. Os tratamentos foram: sala de espera sem climatização (CONTR), sala de espera com ventilação forçada $(V)$ e sala de espera com ventilação forçada e aspersão (VA). As variáveis fisiológicas analisadas foram a temperatura retal (TR), freqüência respiratória (FR) e temperatura de pele (cabeça, dorso e glândula mamária) coletadas antes e depois da aplicação de cada tratamento. As variáveis ambientais registradas foram a temperatura de bulbo seco (TBS), temperatura de globo negro (TGN) e umidade relativa (UR), coletadas antes $e$ depois da aplicação de cada tratamento. O tratamento VA foi mais eficiente em reduzir a TBS $\left(6,4^{\circ} \mathrm{C}\right)$ e $\mathrm{TGN}\left(6,5^{\circ} \mathrm{C}\right)$. Os tratamentos $V$ e VA diminuíram significativamente a FR dos animais. A variável temperatura de pele apresentou redução de $4,2^{\circ} \mathrm{C}$ para a região da cabeça e $2,8^{\circ} \mathrm{C}$ para a região do dorso, no tratamento VA. O tratamento VA proporcionou maiores reduções nas variáveis ambientais e fisiológicas resultando em melhores condições de conforto aos animais e maior eficácia na dissipação de calor pela pele dos animais.

Palavras-chave: estresse térmico, respostas fisiológicas, resfriamento evaporativo.

\section{ABSTRACT}

The aim of this study was to evaluate the effects of climatization (ventilation or ventilation plus sprinkler) inside a holding pen in a milking parlor on environmental and physiological parameters. The experiment was conducted from August to October during 90 consecutive days. Twenty-one lactating dairy cows with an average milk yield of $21 \mathrm{~kg} \mathrm{~d}^{-1}$ were randomly allocated in three treatments. The treatments were: holding pen without cooling (CONTROL); holding pen with ventilation $(V)$ and holding pen with ventilation plus sprinkler (VS). The physiological parameters measured were respiratory rate $(R R)$, body temperature $(B T)$ and skin temperature (head, flank and mammary gland). These measurements were taken before and after each treatment. The data on the environmental parameters dry bulb temperature (DBT), black globe temperature $(B G T)$ and relative humidity $(R H)$ were collected both from each treatment session and from a meteorological station at a nearby site three times a day. Results showed that VS reduced DBT $\left(6.4^{\circ} \mathrm{C}\right)$ and $B G T\left(6.5^{\circ} \mathrm{C}\right)$; and increased the $\mathrm{RH}$. Both V and VS reduced significantly respiratory rate. The skin temperature, with

${ }^{1}$ Projeto financiado pela Fundação de Amparo à Pesquisa do Estado de São Paulo (FAPESP).

${ }^{2}$ Centro de Análise e Pesquisa Tecnológica do Agronegócio (CAPTA), Bovinos de Leite, Instituto de Zootecnia, 13460-000. Nova Odessa, SP, Brasil. E-mail: irineu@apta.sp.gov.br. Autor p/ correspondência.

${ }^{3}$ Genética e Reprodução, IZ, Nova Odessa, SP, Brasil.

${ }^{4}$ Médico Veterinário, Doutorando em Qualidade e Produtividade Animal, Faculdade de Zootecnia e Engenharia de Alimentos, Universidade de São Paulo (FZEA/USP), Brasil.

${ }^{5}$ Zootecnista, Doutorando em Física do Ambiente Agrícola, Núcleo de Pesquisa em Ambiência, Escola Superior de Agricultura "Luiz de Queiroz”, Universidade de São Paulo (ESALQ/USP), Brasil.

${ }^{6}$ Doutorando em Estatística, Universidade Federal de Viçosa (UFV), Brasil. 
VS showed a decrease of $4.2^{\circ} \mathrm{C}$ for the head and $2.8^{\circ} \mathrm{C}$ for the flank. Hence, VS improved the environmental condition and physiological responses and increased heat losses of the dairy cows.

Key words: heat stress, physiological responses, evaporative cooling.

\section{INTRODUÇÃO}

A adoção de modernas tecnologias nos sistemas de produção de leite levou ao aparecimento de animais mais produtivos os quais apresentam metabolismo acelerado, com maior produção de calor endógeno, tornando-se mais susceptíveis aos efeitos do meio ambiente (TITTO, 1998).

Os animais reagem ao estresse térmico com mudanças fisiológicas e comportamentais (BAÊTA et al., 1987). As respostas fisiológicas compreendem o aumento da freqüência respiratória, redução na ingestão de alimentos e aumento na ingestão de água. Como modificação comportamental, as vacas em lactação diminuem o pastejo e ambulação, pastando à noite e buscando sombra e imersão em água durante o dia (PIRES et al., 1998).

Em função da adversidade climática existente entre as regiões, as estratégias de manejo ambientais devem ser adaptadas às condições locais. Um dos grandes problemas da ambiência na produção de leite é que existem vários modelos relacionados aos sistemas de climatização, entretanto, sua maioria, foi desenvolvida em condições ambientais distintas dos países tropicais (MATARAZZO, 2003).

A ventilação desses ambientes pode melhorar as condições termo-higrométricas, ao incrementar as trocas de calor por convecção e evaporação. Assim, a ventilação forçada deve ser adotada sempre que os meios naturais não proporcionarem os índices de renovação de ar necessária (STOWELL et al., 2003).

Em algumas situações, apenas o emprego de ventiladores não é suficiente para alcançar as condições ideais mínimas de conforto, havendo a necessidade de recorrer ao uso do resfriamento adiabático evaporativo (NÄÄS, 1998). Nesses sistemas, a água deve penetrar e umedecer completamente a pele e o pêlo dos animais, de forma que as vacas sejam resfriadas e troquem calor por condução e por evaporação da água, a partir dos pêlos e da pele (BACCARI JR, 2001).
Estudos realizados com vacas holandesas alojadas em instalação parcialmente fechada, localizada no Vale do Pó (Itália), apontaram que a ventilação na velocidade de $0,5 \mathrm{~ms}^{-1}$ reduziu os efeitos negativos do estresse térmico. Quando a ventilação foi associada à aspersão, ocorreu diminuição na taxa respiratória, aumento de 10 a $15 \%$ na umidade relativa e redução de 2,5 a $3,0^{\circ} \mathrm{C}$ na temperatura ambiente, concluindo que a utilização de ventiladores e aspersores foi eficiente em reduzir o estresse térmico (FRAZZI et al., 1997).

Os objetivos do presente trabalho foram avaliar, por meio do índice de temperatura e umidade (ITU) e do índice de globo negro e umidade (IGNU), os efeitos da climatização na sala de espera sobre as respostas fisiológicas (freqüência respiratória, temperatura retal e de pele) de vacas em lactação.

\section{MATERIAL E MÉTODOS}

O presente ensaio foi conduzido na Estação Experimental de Zootecnia, localizada no município de Nova Odessa, SP, com latitude $22^{0}$ $42^{\prime} \mathrm{S}$ e longitude $47^{0} 18^{\prime} \mathrm{W}$ e altitude de $550 \mathrm{~m}$. Foram utilizadas 21 vacas Holandesas, com peso médio de $600 \mathrm{~kg}$ e produção de leite média de 21 $\mathrm{kg} \mathrm{dia}^{-1}$, aos $60 \pm 15$ dias em lactação, durante os meses de agosto, setembro e outubro de 2001. As vacas receberam como alimentação silagem de sorgo, farelo de soja, grãos de milho moído, caroço de algodão e minerais, fornecidos na forma de dieta completa e de maneira a atender às exigências nutricionais para a manutenção e produção de leite, de acordo com o NRC (1989).

Os sete animais foram distribuídos aleatoriamente no três tratamentos a seguir: sala de espera sem climatização (CONTROLE), sala de espera com ventilação forçada (V) ou sala de espera com ventilação e aspersão (VA). Os animais do tratamento $\mathrm{V}$ permaneceram na sala de espera por $30 \mathrm{~min}$ com os ventiladores ligados, sendo posteriormente, ordenhados. Por último, foram ligados os ventiladores e aspersores para o lote correspondente ao tratamento VA que permaneceram por $30 \mathrm{~min}$ na sala de espera e posteriormente foram ordenhados. A ordenha era realizada três vezes ao dia $(7,14$ e $21 \mathrm{~h})$.

Os equipamentos de climatização na sala de espera foram acionados após a ordenha dos animais controle quando a temperatura ambiente era igual ou superior a $25^{\circ} \mathrm{C}$. O sistema 
de ventilação era composto por dois ventiladores, tipo tufão, equipado com motor de $1 / 2 \mathrm{CV}$ e com capacidade de produzir movimentação do ar de até $5 \mathrm{~m} \mathrm{~s}^{-1}$. O sistema de aspersão era de cano PVC com bicos de cerâmica espaçados a $1 \mathrm{~m}$ acionado por moto bomba de $1,5 \mathrm{cv}$.

A freqüência respiratória (contagem dos movimentos do flanco), temperatura retal (termômetro clínico digital) e a temperatura da pele determinada na cabeça, dorso e glândula mamária (termômetro de infravermelho) foram mensuradas diariamente, sempre nos mesmos três animais, antes e depois da aplicação de cada tratamento.

Os registros da temperatura, umidade relativa do ar e da temperatura de globo negro, foram feitos, respectivamente, com psicrômetros e termômetro de globo negro instalados na sala de espera. Os equipamentos foram alocados no centro geométrico da instalação a $1,8 \mathrm{~m}$ de altura do solo. Os dados foram registrados durante a permanência dos animais nesse ambiente, atendendo às condições dos tratamentos (antes e após os equipamentos serem acionados). Um abrigo meteorológico foi colocado no piquete onde os animais permaneceram e, diariamente às 7, 13 e 17h, foram anotados os valores das temperaturas de bulbo seco, bulbo úmido, globo negro, máximas e mínimas e a umidade relativa do ar. A partir dos valores encontrados para as variáveis ambientais foram determinados os índices de conforto térmico índice de temperatura e umidade (ITU) proposto por JOHNSON (1980) e índice de globo negro e umidade (IGNU) desenvolvido por BUFFINGTON et al. (1981). As variáveis ambientais foram arranjadas em esquema fatorial 3 x 2 em delineamento inteiramente casualisado sendo considerado três ambientes
(CONTROLE, V ou VA) e duas medições (antes e depois de aplicado os tratamentos). A análise estatística dos dados foi realizada no programa estatístico SAS (1990).

\section{RESULTADOS E DISCUSSÃO}

Os equipamentos foram acionados somente às $14 \mathrm{~h}$ quando a temperatura média de bulbo seco atingiu os $26,1^{\circ} \mathrm{C}$. No mesmo horário, a temperatura do globo negro foi $26,0^{\circ} \mathrm{C}$ e a umidade relativa do ar de 53,2\%. Os valores médios verificados para as variáveis ambientais e índices de conforto térmico na sala de espera antes e após a aplicação dos tratamentos (CONTROLE, $\mathrm{V}$ ou VA) encontram-se na tabela 1 . O tratamento $\mathrm{V}$ proporcionou aumento da $\mathrm{TBS}\left(1,8^{\circ} \mathrm{C}\right)$ e TGN $\left(1,3^{\circ} \mathrm{C}\right)$ e decréscimo no valor da UR $(7,0 \%)$. O tratamento VA mostrou-se eficiente, reduzindo a TBS $\left(6,4^{\circ} \mathrm{C}\right)$ e $\mathrm{TGN}\left(6,5^{\circ} \mathrm{C}\right)$ e, como esperado, proporcionou aumento na umidade relativa do ar.

Existe uma grande variação na literatura sobre as temperaturas crítica superior e inferior. HUBER (1990) citou a faixa de $4-26^{\circ} \mathrm{C}$ como de conforto para vacas em lactação. De acordo com os resultados encontrados no presente experimento, verificou-se que o tratamento VA proporcionou condições satisfatórias para a produção animal. O sistema de resfriamento adiabático evaporativo (SRAE) consiste em mudar o ponto de estado psicrométrico do ar, para maior umidade e menor temperatura, mediante o contato do ar com uma superfície umedecida ou líquida (WIERSMA et al., 1983). Entretanto, a eficiência do SRAE depende da diferença entre as temperaturas bulbo seco e úmido (depressão psicrométrica) a qual é típica de cada região. O

Tabela 1 - Valores médios da temperatura do bulbo seco (TBS), umidade relativa (UR), temperatura de globo negro (TGN) e índices de cc térmico (ITU e IGNU) encontrados na sala de espera antes e depois da aplicação dos tratamentos no horário das 14 horas.

\begin{tabular}{|c|c|c|c|c|c|c|}
\hline \multirow{3}{*}{ Variáveis } & \multicolumn{6}{|c|}{ Tratamentos } \\
\hline & \multicolumn{2}{|l|}{ CONTROLE } & \multicolumn{2}{|c|}{$\mathrm{V}$} & \multicolumn{2}{|c|}{ VA } \\
\hline & Antes & Depois & Antes & Depois & Antes & Depois \\
\hline$\overline{\mathrm{TBS}},{ }^{\circ} \mathrm{C}$ & $27,4 \pm 2,1 b$ & $27,5 \pm 2,0 \mathrm{~b}$ & $27,5 \pm 2,1 \mathrm{~b}$ & $29,3 \pm 2,4 a$ & $29,3 \pm 2,4$ a & $22,9 \pm 2,0 \mathrm{c}$ \\
\hline UR, \% & $45,7 \pm 15,2$ a & $45,6 \pm 15,4 b$ & $45,9 \pm 15,6 \mathrm{a}$ & $38,9 \pm 13,8 \mathrm{c}$ & $38,9 \pm 13,5 b$ & $79,8 \pm 16,0$ a \\
\hline $\mathrm{TGN},{ }^{\circ} \mathrm{C}$ & $27,4 \pm 2,5 b$ & $27,6 \pm 2,5 b$ & $27,7 \pm 2,6 \mathrm{~b}$ & $29,0 \pm 2,3$ a & $29,1 \pm 2,3$ a & $22,5 \pm 2,3 \mathrm{c}$ \\
\hline ITU & $73,1 \pm 2,1$ b & $73,3 \pm 2,0 \mathrm{~b}$ & $73,4 \pm 2,0 \mathrm{~b}$ & $74,9 \pm 2,2$ a & $74,9 \pm 2,2 \mathrm{a}$ & $70,2 \pm 2,5 \mathrm{c}$ \\
\hline IGNU & $73,5 \pm 2,6$ b & $73,7 \pm 2,5 b$ & $73,8 \pm 2,5$ b & $74,8 \pm 2,2 \mathrm{a}$ & $74,8 \pm 2,2 \mathrm{a}$ & $70,1 \pm 2,1 \mathrm{c}$ \\
\hline
\end{tabular}

(*) Médias seguidas da mesma letra nas linhas não diferem pelo teste de Duncan a 5\% de probabilidade. V - ventilação forçada; VA - ventilação forçada e asperção. 
uso do SRAE permitiu uma queda de 4 a $7^{\circ} \mathrm{C}$ na temperatura, em região dos Estados Unidos, que correspondia a uma depressão psicrométrica (TbsTbu) ao meio dia de 7 e $9^{\circ} \mathrm{C}$. A depressão psicrométrica verificada nas presentes condições foi $10,2^{\circ} \mathrm{C}$ às 14 horas, garantindo a eficiência do tratamento VA.

Considerando-se o THI no horário das 14 horas, verificou-se que, nesse horário, os animais estavam sujeitos ao estresse térmico, uma vez que o valor de THI entre 71 e 78 caracteriza uma condição crítica para os animais (HAHN, 1985). O tratamento VA reduziu o THI para 70,2 proporcionando condições adequadas de conforto térmico para as vacas em lactação. Os valores de BGHI até 74 definem condição de conforto para os bovinos; entre 75 e 78, a situação é de alerta, 79 a 84 caracterizam perigo e, acima deste, deparase com situação de emergência (BAÊTA et al., 1987). De maneira semelhante ao verificado para o THI, o tratamento VA reduziu o BGHI para 70,1 proporcionando um microclima interno satisfatório para o desempenho dos animais.

Os valores médios da temperatura retal (TR), freqüência respiratória (FR) e temperatura de pele dos animais submetidos aos diferentes tratamentos encontram-se na tabela 2. A temperatura retal é usada freqüentemente como índice de adaptabilidade fisiológica em ambientes quentes, pois seu aumento mostra que os mecanismos de liberação de calor tornaram-se insuficientes para manter a homeotermia (MOTA, 1997). A temperatura retal permaneceu dentro dos valores fisiológicos normais $\left(38\right.$ a $39^{\circ} \mathrm{C}$ ) sugeridos por DU PEREZ (2000) em todos os tratamentos. Entretanto, trabalhos conduzidos por TURNER et al. (1992) constataram decréscimo na temperatura retal de animais submetidos ao SRAE.
Os resultados encontrados a FR indicaram que tal variável permaneceu dentro da faixa de normalidade, entretanto, a aplicação dos tratamentos V (37mov min ${ }^{-1}$ ) e VA (38mov min ${ }^{-1}$ ) proporcionaram decréscimos nessa variável. Observações semelhantes foram encontrados por FRAZZI et al. (1997) que verificaram diminuição da freqüência respiratória, sem alteração da temperatura retal quando se utilizou ventilação mais aspersão. Em condições termoneutras, a FR normal da vaca leiteira varia de 24 a 36 movimentos por minuto (STOBER, 1993). A temperatura de pele tomada da região da cabeça, do dorso e da glândula mamária, foi menor nas vacas submetidas ao tratamento VA quando comparada aos outros tratamentos.

A região da cabeça apresentou diminuição média de $4,2^{\circ} \mathrm{C}$. Na região do dorso essa diminuição foi de $2,8^{\circ} \mathrm{C}$ e, na glândula mamária foi de apenas $1,0^{\circ} \mathrm{C}$. Segundo BACCARI JR. (2001) quando a temperatura da pele é mais elevada que a do ambiente, o organismo cede calor às moléculas de ar, dando início à troca térmica. Portanto, quando a temperatura do ambiente onde a vaca se encontra diminui, o animal dissipará mais calor, resfriando a sua temperatura corporal, o que explica a diminuição da temperatura da cabeça, dorso e glândula mamária verificada nos animais submetidos ao tratamento VA.

\section{CONCLUSÕES}

O tratamento ventilação associado à aspersão é eficiente para o condicionamento térmico da sala de espera, uma vez que mantém as variáveis ambientais e fisiológicas dentro da faixa de conforto térmico para as vacas em lactação.

Tabela 2 - Valores médios de temperatura retal (TR), freqüência respiratória (FR) e temperatura de pele (cabeça, dorso e glândula mamária) dos animais no horário das 14 horas.

\begin{tabular}{|c|c|c|c|c|c|c|}
\hline \multirow{3}{*}{ Variáveis } & \multicolumn{6}{|c|}{ Tratamentos } \\
\hline & \multicolumn{2}{|l|}{ CONTROLE } & \multicolumn{2}{|c|}{ V } & \multicolumn{2}{|c|}{ V } \\
\hline & Antes & Depois & Antes & Depois & Antes & Depois \\
\hline $\mathrm{TR},{ }^{\circ} \mathrm{C}$ & $37,8 \pm 0,2$ a & $37,8 \pm 0,2$ a & $38,1 \pm 0,2$ a & $38,1 \pm 0,2$ a & $38,2 \pm 0,2$ a & $38,2 \pm 0,2$ a \\
\hline FR, mov $\min ^{-1}$ & $47,0 \pm 4,5 \mathrm{a}$ & $48,0 \pm 4,5$ a & $42,0 \pm 4,5 \mathrm{~b}$ & $37,0 \pm 4,5$ b & $42,0 \pm 4,5 \mathrm{~b}$ & $38,0 \pm 4,5 \mathrm{~b}$ \\
\hline Cabeça, ${ }^{\circ} \mathrm{C}$ & $31,2 \pm 2,5 a$ & $31,2 \pm 2,5 \mathrm{a}$ & $31,3 \pm 2,5 \mathrm{a}$ & $30,8 \pm 2,5 \mathrm{a}$ & $30,8 \pm 2,5 \mathrm{a}$ & $26,6 \pm 2,5 b$ \\
\hline Dorso, ${ }^{\circ} \mathrm{C}$ & $33,5 \pm 1,0 \mathrm{a}$ & $33,5 \pm 1,0 \mathrm{a}$ & $33,7 \pm 1,0 \mathrm{a}$ & $33,1 \pm 1,0 \mathrm{a}$ & $33,4 \pm a$ & $30,6 \pm 1,0 \mathrm{~b}$ \\
\hline Gl. mamária, ${ }^{\circ} \mathrm{C}$ & $33,8 \pm 0,5 c$ & $33,8 \pm 0,5 c$ & $34,7 \pm 0,5 a$ & $34,2 \pm 0,5 \mathrm{a}$ & $34,3 \pm 0,5 b$ & $33,3 \pm 0,5 c$ \\
\hline
\end{tabular}

$(*)$ Médias seguidas da mesma letra nas linhas não diferem pelo teste de Duncan a 5\% de probabilidade.

V - ventilação forçada; VA - ventilação forçada e asperção.

Ciência Rural, v.35, n.3, mai-jun, 2005. 


\section{REFERÊNCIAS BIBLIOGRÁFICAS}

BACCARI JR. F. Manejo ambiental da vaca leiteira em climas quentes. Londrina : UEL, 2001. 142p.

BAÊTA, F.C. et al. Equivalent temperature index at temperatures above the thermoneutral for lactating cows. Transactions of the ASAE, Paper n.8874015, p.1-21, 1987.

BUFFINGTON, D.E. et al. Black globe-humidity index (BGHI) as comfort equation for dairy cows. Transactions of the ASAE, v.24, p.711-714, 1981.

DU PREEZ, J.H. Parameters for the determination and evaluation of heat stress in dairy cattle in South Africa. Onderstepoort Journal Veterinary Research, v.67, p.263-271, 2000.

FRAZZI, E. et al. The aeration, with and without misting: effects on heat stress in dairy cows. Minnesota, 1997. In: INTERNATIONAL SYMPOSIUM LIVESTOCK ENVIRONMENT, 5., 1997, Minnesota. Proceedings... Minnesota : ASAE, 1997. p.907-914.

HAHN, G.L. Compensatory performance in livestock: influences. In: YOUSEF, M.K. Stress physiology. Воса Raton : CRC, 1985. V.2, p.52-145.

HUBER, J.T. Alimentação de vacas de alta produção sob condições de estresse térmico. Piracicaba, SP, 1990. In: BOVINOCUltURA LEITEIRA, 1990, Piracicaba. Anais... Piracicaba : FEALQ, 1990. p.33-48.

JOHNSON, H.D. Environmental management of cattle to minimize the stress of climatic change. International Journal Biometeorology, v.24, p.65-78, 1980.

MATARAZZO, S.V. et al. Intermitência de acionamento do sistema de resfriamento evaporativo em freestall e sua influencia no conforto térmico de vacas em lactação. In: REUNIÃO DA SOCIEDADE BRASILEIRA DE ZOOTECNIA, 40., 2003, Santa Maria. Anais... Santa Maria : UFSM, 2003. CD-room.

MOTA, L.S. Adaptação e interação genótipo-ambiente em vacas leiteiras. 1997. 69f. Tese (Doutorado em genética) - Faculdade de Medicina de Ribeirão Preto, Universidade de São Paulo.

NÄÄS, I.A. Tipologia de instalações em clima quente. Piracicaba, SP, 1998. In: SIMPÓSIO BRASILEIRO DE AMBIÊNCIA NA PRODUÇÃO DE LEITE, 1., 1998, Piracicaba, SP. Anais... Piracicaba : FEALQ, 1998. p.146155.

NRC. Nutrient requeriments of dairy cattle. Washington: National Academy, 1989. 158p.

PIRES, M.F.A. et al. Reflexos do estresse térmico no comportamento das vacas em lactação. Piracicaba, SP, 1998. In: SIMPÓSIO BRASILEIRO DE AMBIÊNCIA NA PRODUÇÃO DE LEITE, 1., 1998, Piracicaba, SP. Anais... Piracicaba : FEALQ, 1998. p.68-102.

SAS. User's guide: Statistics (Release 6,03). Cary : SAS Institute, 1990. 620p.

STOBER, M. Identificação, anamnese, regras básicas da técnica do exame clínico geral. In: ROSEMBERG (Ed). Exame clínico dos bovinos. Rio de Janeiro : Guanabara Koogan, 1993. 419p.

STOWELL, R.R. et al. Design parameters for hotweather ventilation of dairy housing: a critical review. Forth Worth, Tx, 2003. In: INTERNATIONAL DAIRY HOUSING, 5., 2003, Forth Worth, Tx. Proceedings... St. Joseph : ASAE, 2003. CD-room.

TITTO, E.A.L. Clima:influência na produção de leite. Piracicaba, SP, 1998. In: SIMPÓSIO BRASILEIRO DE AMBIÊNCIA NA PRODUÇÃO DE LEITE, 1., 1998, Piracicaba, SP. Anais... Piracicaba : FEALQ, 1998. p.1023.

TURNER, L.W. et al. Reducing heat stress in dairy cows through sprinkler and fan cooling. Applied Engineering in Agriculture. v.8, n.3, p.375-379, 1992.

WIERSMA, F. et al. Evaporative cooling. In: HELLICKSON, M. A. et al. Ventilation of agricultural structures. St. Joseph : ASAE, 1983. p.113-118. 